\title{
Desenvolvimento e exigências térmicas de Orius insidiosus (Say) (Hemiptera, Anthocoridae)
}

\author{
Simone Martins Mendes ${ }^{1}$, Vanda Helena Paes Bueno ${ }^{1} \&$ Lívia Mendes Carvalho $^{1}$
}

'Departamento de Entomologia, Universidade Federal de Lavras, Caixa Postal 3037, 37200-000, Lavras-MG, Brasil.
mmsimone@brfree.com.br; vhpbueno@ufla.br

\begin{abstract}
Development and thermal requirements of Orius insidiosus (Say) (Hemiptera: Anthocoridae). The temperature strongly influences the development time of insects and the understanding of this aspect for natural enemies is essential for its use as biocontrol agents and for mass rearing purpose. The objective of this work was evaluating the effect of different temperatures on the development time of Orius insidiosus (Say, 1832) as well as its thermal requirements. The trials were conducted in climatic chamber at 16, 19, 22, 25, 28 and $31 \pm 1^{\circ} \mathrm{C}$; RH $70 \pm 10 \%$ and photophase 12h. Eggs of Anagasta kuehniella (Zeller, 1879) were provided as food. The embryonic period was 14.0, $8.9,6.6,4.8,3.9$ and 3.3 days at $16,19,22,25,28$ and $31^{\circ} \mathrm{C}$, respectively. Nymphs of all instars (independent of originated sex) were affected by the temperature on their development time; there was a reduction of this period with the increase of the temperature. Males and females presented a development time about 12 days at $25^{\circ} \mathrm{C}$. The developmental thresholds $\left(\mathrm{T}_{\mathrm{o}}\right)$ for egg stage was estimated at $11.78^{\circ} \mathrm{C}$, and for the nymphal phase were $12.27^{\circ} \mathrm{C}$ and $13.03^{\circ} \mathrm{C}$ for males and females, respectively. The thermal constants $(\mathrm{K})$ for egg stage (63.75 day-degrees) and for nymphal stage were 161.97 and 157.24 day-degrees, for males and females, respectively. The temperature of $25^{\circ} \mathrm{C}$ was the most suitable for development time of $O$. insidiosus.
\end{abstract}

KEYWORDS. Biological control; Orius spp.; predator; temperature.

RESUMO. Desenvolvimento e exigências térmicas de Orius insidiosus (Say) (Hemiptera, Anthocoridae). A temperatura exerce grande influência no desenvolvimento dos insetos e o conhecimento desse aspecto é essencial para subsidiar o uso de inimigos naturais como agentes de controle biológico, bem como para a sua criação massal. O presente trabalho teve como objetivo avaliar o efeito de diferentes temperaturas no desenvolvimento de Orius insidiosus (Say, 1832), bem como as suas exigências térmicas. O experimento foi conduzido em câmaras climáticas, a $16,19,22,25,28$ e $31 \pm 1^{\circ} \mathrm{C}$; UR de $70 \pm 10 \%$ e fotofase de 12 horas. Como alimento foram utilizados ovos de Anagasta kuehniella (Zeller, 1879). O período embrionário foi de 14,$0 ; 8,9 ; 6,6 ; 4,8 ; 3,9$ e 3,3 dias nas temperaturas de $16,19,22,25,28$ e $31^{\circ} \mathrm{C}$, respectivamente. Ninfas de todos os instares (independente do sexo que deram origem) foram influenciadas pela temperatura quanto ao seu desenvolvimento, com redução nesse período com o aumento da temperatura. Machos e fêmeas, na temperatura de $25^{\circ} \mathrm{C}$, apresentaram um período de desenvolvimento em torno de 12 dias. A temperatura base da fase de ovo foi de $11,78^{\circ} \mathrm{C}$ e a da fase ninfal foi de $12,27^{\circ} \mathrm{C}$ e de $13,03^{\circ} \mathrm{C}$, para machos e fêmeas, respectivamente. A constante térmica para a fase de ovo foi de 63,75 e para a fase de ninfa de 161,97 e 157,24 graus-dia, para machos e fêmeas, respectivamente. A temperatura de $25^{\circ} \mathrm{C}$ foi a mais adequada para o desenvolvimento de $O$. insidiosus.

PALAVRAS-CHAVE. Controle biológico; Orius spp.; predador; temperatura.

Percevejos antocorídeos do gênero Orius são amplamente distribuídos pelas diferentes regiões zoogeográficas e vários fatores ecológicos podem influenciar as diversas características biológicas da maioria das espécies desse gênero, entre eles a temperatura, o fotoperíodo e o tipo de presa (Isenhour \& Yeargan, 1981; Riudavets, 1995; Lattin, 1999; Argolo, 2000; Mendes et al., 2002).

A espécie Orius insidiosus (Say, 1832) tem origem neártica e, de acordo com Ruberson et al. (1996), a maior parte dos predadores heterópteros, em regiões de clima temperado, pode passar o inverno, período de baixas temperaturas e fotoperíodo curto, nos diferentes estágios de seu ciclo biológico, mas a maioria das espécies estudadas passa esse período na fase adulta.

O desenvolvimento desses insetos é influenciado pela temperatura. Van Den Meiracker (1999) observou haver uma diminuição no desenvolvimento de $O$. insidiosus quando a temperatura variou entre 18 e $30^{\circ} \mathrm{C}$. Isenhour \& Yeargan (1981) verificaram um período de desenvolvimento de 20 e 12,7 dias, a 24 e $28^{\circ} \mathrm{C}$, respectivamente para este mesmo predador, presente em regiões temperadas.

Higley et al. (1986) mencionaram que altas temperaturas ativam o funcionamento de enzimas, aumentam a velocidade de reações químicas e, conseqüentemente, diminuem o tempo de desenvolvimento; isso ocorre, obviamente, dentro de uma faixa inerente a cada espécie. Também, segundo Dent (1997), para saber a quantidade de calor requerida para um inseto completar seu desenvolvimento ou um estágio, é necessário o conhecimento da temperatura base de um estágio particular desse inseto e a sua taxa de desenvolvimento em relação à temperatura.

No entanto, respostas à temperatura não estão bem elucidadas para as espécies de Orius presentes em regiões tropicais, principalmente para $O$. insidiosus, que é a espécie mais comum nos agroecosistemas do Brasil (Bueno, 2000).

É notório também que os fatores ecológicos atuam em conjunto. Contudo, o isolamento de um fator em condições de laboratório também fornece dados importantes no contexto 
do desenvolvimento dos insetos. Particularmente para $O$. insidiosus, esses dados poderão auxiliar na otimização de sua criação massal, bem como no estabelecimento em áreas onde possa ser liberado e/ou introduzido como agente de controle biológico.

Este trabalho teve como objetivos avaliar a influência de diferentes temperaturas no desenvolvimento e sobrevivência de O. insidiosus tendo ovos de Anagasta kuehniella (Zeller, 1879) como alimento, bem como determinar as exigências térmicas desse predador.

\section{MATERIAL E MÉTODOS}

O experimento foi conduzido no Laboratório de Controle Biológico do Departamento de Entomologia da Universidade Federal de Lavras, onde é mantida criação de pesquisa do predador $O$. insidiosus de acordo com a metodologia proposta por Bueno (2000) e Mendes \& Bueno (2001).

Desenvolvimento de $\boldsymbol{O}$. insidiosus em diferentes temperaturas: Estudos do desenvolvimento do predador foram realizados em câmaras climatizadas, nas temperaturas de $16,19,22,25,28$ e $31 \pm 1^{\circ} \mathrm{C}$ (são temperaturas que normalmente ocorrem em sistemas de cultivos protegidos), com umidade relativa de $70 \pm 10 \%$ e fotofase de 12 horas.

Da criação de pesquisa foram retirados ovos com até 24 horas de idade, da $3^{\mathrm{a}}$ geração de laboratório (por questões de padronização), depositados pelas fêmeas em inflorescências de picão-preto, Bidens pilosa L. (Asteraceae). Os ovos foram mantidos sob as diferentes temperaturas avaliadas e diariamente observados, quanto à eclosão das ninfas.

Ninfas recém-eclodidas foram colocadas em placas de petri ( $5 \mathrm{~cm}$ de diâmetro) contendo um pedaço de algodão (aproximadamente $1 \mathrm{~cm}^{2}$ ) umedecido com água destilada, um pedaço de papel sulfite branco $\left(0,5 \mathrm{~cm}^{2}\right)$ e ovos de $A$. kuehniella ad libitum como presa. Diariamente, as ninfas foram observadas quanto à mudança de instar (presença de exúvia), ocasião em que também eram fornecidos alimento e água.

Foram avaliados, nas diferentes temperaturas, o período embrionário, as durações de cada instar e da fase ninfal, de ninfas que deram origem a machos e fêmeas e a sobrevivência de cada estádio de desenvolvimento do predador. O delineamento experimental foi inteiramente ao acaso, composto de seis temperaturas com número diferente de repetições por tratamento. Para avaliação da sobrevivência foi feita análise da interação instar do predador e temperatura. Os dados de todos os parâmetros avaliados foram submetidos à análise de variância e as médias comparadas pelo teste de Scott \& Knott $(\mathrm{P} \leq 0,01)$ (Scott \& Knott, 1974).

Exigências térmicas de $\boldsymbol{O}$. insidiosus: Foram determinadas as exigências térmicas de $O$. insidiosus quanto ao limite térmico inferior de desenvolvimento ou temperatura base (Tb) e a constante térmica (K), expressa em graus-dia (GD), calculadas pelo método da hipérbole e sua recíproca, de acordo com método da hipérbole utilizado por Haddad \& Parra (1984).

\section{RESULTADOS E DISCUSSÃO}

Período embrionário: $\mathrm{O}$ período embrionário de $O$. insidiosus apresentou diferenças significativas em todas as temperaturas avaliadas, acentuando uma diminuição com o aumento da temperatura de 16 para $31^{\circ} \mathrm{C}$. A diferença observada nesse período na menor temperatura $\left(16^{\circ} \mathrm{C}\right.$ com duração de 14 dias) para a maior $\left(31^{\circ} \mathrm{C}\right.$ com duração de 3,3 dias), foi cerca de 4 vezes (Tabela I), evidenciando influência direta do aumento da temperatura no incremento do metabolismo do embrião em desenvolvimento. Segundo Dent (1997), a taxa de desenvolvimento de um inseto é primariamente dependente da temperatura.

Esses resultados assemelham-se aos de Mendes \& Bueno (2001), os quais encontraram 5,3 dias para o período embrionário de O. insidiosus a $25^{\circ} \mathrm{C}$. Também Mccffrey \& Horsburg (1986) observaram 11,6 dias para esse período na temperatura de $17^{\circ} \mathrm{C}$ e 3,1 dias quando $O$. insidiosus foi exposto a $35^{\circ} \mathrm{C}$. Já Isenhour \& Yeargan (1981) obtiveram 8,8; 5,1; 3,9 e 3,5 dias nas temperaturas de $20,24,28$ e $32^{\circ} \mathrm{C}$, respectivamente.

Desenvolvimento e sobrevivência ninfal de $O$. insidiosus: $\mathrm{O}$ desenvolvimento ninfal de $O$. insidiosus foi dependente da temperatura, ocorrendo diferenças significativas na duração de todos os estádios para ambos os sexos do predador (Tabela I).

A duração do $1^{\circ}, 2^{\circ}$ e $3^{\circ}$ ínstares em relação à temperatura mais alta $\left(31^{\circ} \mathrm{C}\right)$ foi cerca de quatro vezes mais curta quando comparada àquela na temperatura mais baixa $\left(16^{\circ} \mathrm{C}\right)$ (Tabela I). Esse aumento na velocidade de desenvolvimento foi ainda maior no intervalo de 16 para $31^{\circ} \mathrm{C}$, para os $4^{\circ}$ e $5^{\circ}$ ínstares, sendo cerca de cinco vezes mais rápido a $31^{\circ} \mathrm{C}$. Este fato, de acordo com Higley et al. (1986), ocorre em função da aceleração na velocidade de reações químicas, que acontece com o aumento da temperatura, independente do sexo.

O $5^{\circ}$ ínstar de $O$. insidiosus apresentou maior duração do que os demais ínstares (Tabela I). Este fato é considerado comum entre predadores do gênero Orius, sendo também verificado para a mesma espécie de predador por Isenhour \& Yeargan (1981), Mccffrey \& Horsburg (1986) e Mendes \& Bueno (2001), e também para outras espécies, como Orius strigicollis (Poppius) (Otha, 2002), Orius majusculus (Reuter, 1879), Orius niger (Wolff, 1811) e Orius laevigatus (Fieber, 1860) (Tommasini \& Nicoli, 1994).

Nas temperaturas mais altas $\left(28\right.$ e $\left.31^{\circ} \mathrm{C}\right)$, houve uma tendência de estabilização no período de desenvolvimento dos diferentes estádios, bem como do período ninfal de $O$. insidiosus (Tabela I). Resultado semelhante foi observado por Isenhour \& Yeargan (1981), que encontraram um desenvolvimento ninfal de 12 dias entre 28 e $32^{\circ} \mathrm{C}$, também utilizando ovos de lepidópteros como presa.

Assim, pode-se inferir que não só a temperatura, mas o tipo de alimento e o fotoperíodo são também fatores que podem influenciar o desenvolvimento ninfal de $O$. insidiosus, podendo seu comportamento, em condições de campo, ser um reflexo do efeito conjunto desses vários fatores. Mendes et al. (2002) encontraram diferenças significativas nesse 
Tabela I. Desenvolvimento (dias) para machos e fêmeas de Orius insidiosus (Say), em diferentes temperaturas, UR $70 \pm 10 \%$ e fotofase de 12 horas.

\begin{tabular}{|c|c|c|c|c|c|c|c|}
\hline \multirow{2}{*}{\multicolumn{2}{|c|}{ Estágio/sexo }} & \multicolumn{6}{|c|}{ Temperatura $\left({ }^{\circ} \mathrm{C}\right)$} \\
\hline & & 16 & 19 & 22 & 25 & 28 & 31 \\
\hline período emb & rionário & $14,0 \pm 0,12 \mathrm{fn}=334$ & $8,9 \pm 0,04$ en $=570$ & $6,6 \pm 0,03 \mathrm{dn}=380$ & $4,8 \pm 0,03 \mathrm{cn}=530$ & $3,9 \pm 0,03 \mathrm{bn}=590$ & $3,3 \pm 0,03 \mathrm{an}=560$ \\
\hline \multirow{2}{*}{$1^{\circ}$ ínstar } & Macho & $8,9 \pm 0,28 \mathrm{en}=27$ & $4,9 \pm 0,18 \mathrm{dn}=34$ & $3,4 \pm 0,10 \mathrm{cn}=44$ & $2,1 \pm 0,12 b n=32$ & $1,9 \pm 0,13 \mathrm{an}=52$ & $1,9 \pm 0,06 \mathrm{an}=35$ \\
\hline & Fêmea & $8,8 \pm 0,34$ en $=23$ & $4,8 \pm 0,15 \mathrm{dn}=26$ & $3,3 \pm 0,09 \mathrm{cn}=52$ & $2,1 \pm 0,08 \mathrm{bn}=40$ & $1,8 \pm 0,08$ an $=37$ & $1,9 \pm 0,05 \mathrm{bn}=49$ \\
\hline \multirow{2}{*}{$2^{\circ}$ ínstar } & Macho & $6,7 \pm 0,24$ en $=27$ & $4,0 \pm 0,13 \mathrm{dn}=34$ & $2,7 \pm 0,10 \mathrm{cn}=44$ & $2,1 \pm 0,78 b n=32$ & $1,6 \pm 0,07 \mathrm{an}=51$ & $1,6 \pm 0,09 \mathrm{an}=34$ \\
\hline & & & & & & & \\
\hline \multirow{2}{*}{$3^{\circ}$ ínstar } & Macho & & & $2,6 \pm 0,11 \mathrm{cn}=40$ & $9 \pm 0,08 \mathrm{bn}=57$ & $1,4 \pm 0,08 \mathrm{sn}=45$ & $1,5 \pm 0,08 \mathrm{bn}=35$ \\
\hline & Fêmea & $6,7 \pm 0,07$ en $=45$ & $4,0 \pm 0,10 \mathrm{dn}=26$ & $2,8 \pm 0,08 \mathrm{cn}=50$ & $2,0 \pm 0,10 \mathrm{bn}=40$ & $1,6 \pm 0,10 \mathrm{an}=35$ & $1,5 \pm 0,07 \mathrm{an}=45$ \\
\hline \multirow{2}{*}{$4^{\circ}$ ínstar } & Macho & $7,7 \pm 0,27 \mathrm{fn}=27$ & $4,4 \pm 0,15 \mathrm{en}=30$ & $3,0 \pm 0,09 \mathrm{dn}=40$ & $2,0 \pm 0,10 \mathrm{cn}=32$ & $1,6 \pm 0,08 b n=40$ & $1,4 \pm 0,08$ an $=35$ \\
\hline & Fêmea & $8,0 \pm 0,08 \mathrm{fn}=22$ & $4,3 \pm 0,14 \mathrm{en}=26$ & $2,9 \pm 0,08 \mathrm{dn}=52$ & $2,0 \pm 0,09 \mathrm{cn}=38$ & $1,6 \pm 0,09 \mathrm{bn}=35$ & $1,4 \pm 0,08 \mathrm{an}=35$ \\
\hline \multirow{2}{*}{$5^{\circ}$ ínstar } & Macho & $15,2 \pm 0,5$ en $=25$ & $7,7 \pm 0,25 \mathrm{dn}=30$ & $5,5 \pm 0,11 \mathrm{cn}=40$ & $3,7 \pm 0,11 \mathrm{bn}=30$ & $2,8 \pm 0,09 \mathrm{an}=40$ & $2,9 \pm 0,08 \mathrm{an}=30$ \\
\hline & Fêmea & $14,0 \pm 0,17 \mathrm{fn}=19$ & $7,8 \pm 0,13$ en $=15$ & $5,7 \pm 0,12 \mathrm{dn}=40$ & $3,9 \pm 0,12 \mathrm{cn}=26$ & $2,9 \pm 0,10 \mathrm{bn}=25$ & $2,6 \pm 0,11$ an $=32$ \\
\hline \multirow{2}{*}{ Fase ninfal } & Macho & $45,0 \pm 0,94 \mathrm{en}=27$ & $24,0 \pm 031 \mathrm{dn}=34$ & $17,7 \pm 0,29 \mathrm{cn}=23$ & $12,4 \pm 0,29 b n=23$ & $9,3 \pm 0,15 \mathrm{an}=36$ & $9,3 \pm 0,23 \mathrm{an}=22$ \\
\hline & Fêmea & $44,7 \pm 0,69 \mathrm{en}=22$ & $25,1 \pm 0,56 \mathrm{dn}=25$ & $17,3 \pm 0,17 \mathrm{cn}=25$ & $12,1 \pm 0,19 b n=25$ & $9,6 \pm 0,22 \mathrm{an}=26$ & $8,9 \pm 0,26 \mathrm{an}=25$ \\
\hline
\end{tabular}

*Médias seguidas de mesma letra nas linhas não diferem entre si por Sott \& Knott $(P<0,01)$.

período para ninfas alimentadas com ovos de A. kuehniella (13,1 dias), para aquelas alimentadas com Aphis gossypii (Glover, 1877) (11,2 dias) e para as alimentadas com Caliothrips phaseoli (Hood, 1912) (10,2 dias), a $25^{\circ} \mathrm{C}$. Também Argolo (2000) demonstrou que, sob fotofase de 9 horas, o desenvolvimento desse predador foi de 11,6 dias, com 12 horas, de 15 dias e sob 14 horas de luz de 13,8 dias, sob a mesma temperatura.

O desenvolvimento ninfal, para ambos os sexos de $O$. insidiosus apresentou variação nas diferentes temperaturas a que foi submetido (Tabela I). Machos e fêmeas apresentaram, respectivamente, um desenvolvimento de 45,0 e 44,7 dias a $16^{\circ} \mathrm{C}$ e de 9,3 e 8,9 dias a $31^{\circ} \mathrm{C}$. Esses resultados são semelhantes aos encontrados por Otha (2002), quando avaliou o efeito da temperatura em ninfas, macho e fêmea de $O$. strigicollis. Sob a temperatura de $18^{\circ} \mathrm{C}$, segundo Van Den Meiracker (1999), o desenvolvimento ninfal de $O$. insidiosus foi de 35,0 dias para fêmeas e 34,7 dias para machos, e a $25^{\circ} \mathrm{C}$, o mesmo autor, em 1994, encontrou um período de desenvolvimento ninfal de 13,77 e 13,89 dias em fêmeas e machos, respectivamente. Esse desenvolvimento foi mais longo daquele obtido neste experimento, em que a $25^{\circ} \mathrm{C}$ machos e fêmeas apresentaram um período ninfal em torno de 12 dias, e a $19^{\circ} \mathrm{C}$, de 24 e 25 dias, respectivamente (Tabela I).

Observou-se que as diferentes temperaturas às quais as ninfas de $O$. insidiosus foram submetidas, também influenciaram na coloração do seu corpo. Essas apresentaram coloração mais escura quando se desenvolveram em temperaturas mais baixas, particularmente naquelas mantidas a $16^{\circ} \mathrm{C}$. De acordo com Chapman (1998), a temperatura tem efeito direto na coloração de muitos insetos, sendo comum que aqueles criados em temperaturas mais baixas apresentem o corpo mais escuro do que aqueles mantidos em temperaturas mais altas, os quais são normalmente mais pálidos. Isso ocorre porque cores escuras refletem pouca radiação, conservando mais o calor.

Quanto à sobrevivência, interação estádio de desenvolvimento e temperatura, não foi significativa, contudo, houve efeito desses parâmetros separadamente (Tabela II). O primeiro ínstar de $O$. insidiosus foi o mais influenciado pelas diferentes temperaturas avaliadas, sendo sua sobrevivência menor do que nos demais ínstares, com $76,0 \% ; 83,3 \% ; 79,2 \%$; $83,3 \% ; 70,8 \%$ e $69,0 \%$ de sobrevivência nas temperaturas de $16,19,22,25,28$ e $31^{\circ} \mathrm{C}$, respectivamente (Tabela II). Este fato, provavelmente, ocorreu devido à maior fragilidade e ao pequeno tamanho do primeiro ínstar, sobretudo quando comparado aos demais ínstares (Tabela II). Schmidt et al. (1998) relataram que a dessecação de ovos e de ninfas do primeiro e segundo ínstares é a maior causa de mortalidade nesse predador. Van Den Meiracker (1999) também observou que a mortalidade ninfal de $O$. insidiosus foi sempre restrita ao primeiro ínstar, correspondendo a $92 \%$ da mortalidade ninfal total. Além disso, também a manipulação dos insetos pode causar maior mortalidade nesses estágios.

Grande parte da porcentagem de mortalidade verificada no $5^{\circ}$ ínstar ocorreu com maior intensidade durante a ecdise para a fase adulta (Tabela II), ou seja, as ninfas que não conseguiram completar a sua fase imatura morreram no processo da última ecdise. Também Van Den Meiracker (1999) encontrou cerca de $12 \%$ de mortalidade de ninfas em $5^{\circ}$ ínstar que não conseguiram completar a última ecdise.

Não foi observada influência da temperatura na sobrevivência da fase ninfal de $O$. insidiosus (Tabela II). Contudo, Messenger (1959) afirmou que o mais importante fator bioclimático para o inseto é a relação quantitativa entre temperaturas constantes e como elas afetam o desenvolvimento e sobrevivência do mesmo.

No processo de escolha de uma temperatura adequada para utilização em criações em laboratório, deve-se levar em consideração características quanto ao desenvolvimento e sobrevivência. Os resultados quanto a esses parâmetros, referentes a $O$. insidiosus, indicam que temperaturas acima de $19^{\circ} \mathrm{C}$ e abaixo de $31^{\circ} \mathrm{C}$ são adequadas para o desenvolvimento do predador, observando a sobrevivência das ninfas. Neste 
Tabela II. Percentagem de sobrevivência de cada instar e da fase ninfal de Orius insidiosus (Say) em diferentes temperaturas, UR $70 \pm 10 \%$ e fotofase de 12 horas.

\begin{tabular}{|c|c|c|c|c|c|c|}
\hline \multirow[t]{2}{*}{ Temp. } & \multicolumn{6}{|c|}{ Sobrevivência (\%) } \\
\hline & $1^{\circ}$ instar & $2^{\circ}$ instar & $3^{\circ}$ instar & $4^{\circ}$ instar & $5^{\circ}$ instar & Fase ninfal \\
\hline $16^{\circ} \mathrm{C}$ & $76,0 \pm 4,17 \mathrm{Bb}$ & $89,7 \pm 3,32 \mathrm{Bb}$ & $94,3 \pm 3,98 \mathrm{Aa}$ & $96,9 \pm 3,03 \mathrm{Aa}$ & $90,6 \pm 5,24 \mathrm{Aa}$ & $62,0 \pm 6,93 \mathrm{~A}$ \\
\hline $19^{\circ} \mathrm{C}$ & $83,3 \pm 4,82 \mathrm{Ab}$ & $97,9 \pm 2,59 \mathrm{Aa}$ & $3,5 \pm 3,68 \mathrm{Aa}$ & $93,0 \pm 3,93 \mathrm{Aa}$ & $92,5 \pm 4,22 \mathrm{Aa}$ & $61,7 \pm 6,33 \mathrm{~A}$ \\
\hline $22^{\circ} \mathrm{C}$ & $79,2 \pm 4,46 \mathrm{Ab}$ & $94,9 \pm 2,51 \mathrm{Aa}$ & $95,3 \pm 2,89 \mathrm{Aa}$ & $95,7 \pm 2,44 \mathrm{Aa}$ & $92,5 \pm 3,23 \mathrm{Aa}$ & $62,5 \pm 4,97 \mathrm{~A}$ \\
\hline $25^{\circ} \mathrm{C}$ & $83,3 \pm 5,08 \mathrm{Ab}$ & $92,8 \pm 3,47 \mathrm{Aa}$ & $96,4 \pm 3,74 \mathrm{Aa}$ & $94,2 \pm 3,26 \mathrm{Aa}$ & $94,2 \pm 3,26 \mathrm{Aa}$ & $68,01 \pm 5,5 \mathrm{~A}$ \\
\hline $28^{\circ} \mathrm{C}$ & $70,8 \pm 4,84 \mathrm{Bb}$ & $89,1 \pm 3,93 \mathrm{Ba}$ & $96,5 \pm 2,46 \mathrm{Aa}$ & $96,4 \pm 2,55 \mathrm{Aa}$ & $92,5 \pm 3,66 \mathrm{Aa}$ & $55,0 \pm 5,29 \mathrm{~A}$ \\
\hline $31^{\circ} \mathrm{C}$ & $69,0 \pm 6,12 \mathrm{Bc}$ & $86,4 \pm 4,49 \mathrm{Bb}$ & $94,2 \pm 3,2 \mathrm{Aa}$ & $95,7 \pm 2,97 \mathrm{Aa}$ & $87,2 \pm 4,93 \mathrm{Ab}$ & $50,0 \pm 5,48 \mathrm{~A}$ \\
\hline
\end{tabular}

*Médias seguidas de mesma letra, maiúscula nas colunas e minúsculas nas linhas, não diferem entre si pelo teste de Scott \& Knott $(\mathrm{P}<0,01)$

contexto, as ninfas apresentaram menor período de desenvolvimento a $25^{\circ} \mathrm{C}$ (Tabela I).

Contudo, também foi relatado por Messenger (1959) não é somente a temperatura o fator que favorece a maior velocidade de desenvolvimento e alta sobrevivência do inseto, mas outras características, como as reprodutivas e, estudos de campo, que determinem abundância e constância, devem ser considerados.

Exigências térmicas de $\boldsymbol{O}$. insidiosus: A velocidade de desenvolvimento de $O$. insidiosus, em função da temperatura, ajustou-se ao modelo linear obtido por meio da recíproca da equação da hipérbole para todos os instares, em ambos os sexos (Tabela III e Fig. 1).

A fase ninfal de $O$. insidiosus apresentou uma temperatura base de $12,27^{\circ} \mathrm{C}$ para os machos e de $13,03^{\circ} \mathrm{C}$ para as fêmeas (Tabela III e Fig. 1). Para o estágio de ovo, essa temperatura $\left(11,78^{\circ} \mathrm{C}\right)$ foi mais baixa do que a encontrada para a fase ninfal de ambos sexos (Tabela III e Fig. 1). Esse valor $\left(11,78^{\circ} \mathrm{C}\right)$ foi semelhante ao encontrado por Isenhour \& Yeargan (1981) $\left(11,2^{\circ} \mathrm{C}\right)$ para a fase de ovo do predador, evidenciando que esse estágio é resistente a temperaturas mais baixas do que a fase ninfal de $O$. insidiosus. Tal fato pode ocorrer devido a proteção oferecida pela presença do córion e pela inserção do ovo no tecido da planta (postura endofítica).

A temperatura base para todos os ínstares do predador foi muito semelhante entre si (Tabela III), tanto para ninfas que deram origem a machos quanto para fêmeas, variando de $11,40^{\circ} \mathrm{C}$ a $13,16^{\circ} \mathrm{C}$, sendo esse valores semelhantes aos verificados por Isenhour \& Yeargan (1981), para os ínstares desse predador.

As constantes térmicas obtidas para $O$. insidiosus nas fases de ovo e de ninfa macho e fêmea foram 63,75; 161,97 e 157,24 GD, respectivamente (Tabela III). Essa constante térmica foi semelhante para todos os instares, com exceção do $5^{\circ}$ ínstar, que apresentou maior exigência de graus-dia para o desenvolvimento $(48,49$ GD para o macho e 45,72 GD para a fêmea) (Tabela III), que está de acordo com o mais longo período de desenvolvimento verificado para este ínstar em relação aos demais (Tabela I).

As diferenças obtidas quanto às exigências térmicas para os diferentes estágios/estádios de desenvolvimento de $O$. insidiosus podem ser atribuídas às diferenças estruturais fisiológicas próprias de cada um, as quais garantem à espécie a capacidade de adaptação às variações ambientais e que são características inerentes da espécie.

Para o desenvolvimento ninfal de $O$. insidiosus os valores de temperatura base encontrados $\left(12,27^{\circ} \mathrm{C}\right.$ para macho e $13,03^{\circ} \mathrm{C}$ para fêmea), foram semelhantes ao verificado por Isenhour \& Yeargan (1981), de $13,7^{\circ} \mathrm{C}$ e superiores ao observado por Mccffrey \& Horsburg (1986), de $10,2^{\circ} \mathrm{C}$, para esse mesmo predador presente em regiões temperadas.

Esses dados indicam existir pouca diferença no que tange à temperatura base para essa espécie, tanto para a presente nas regiões temperadas como para aquela presente na região tropical. Entretanto, diferenças podem ocorrer por influência de outros fatores ecológicos envolvidos, como o tipo de presa e o fotoperíodo, assim como a ocorrência de "raças" de $O$. insidiosus, as quais apresentam características próprias quanto à adaptação a diferentes áreas geográficas.

Também a temperatura base da fase ninfal de $O$. insidiosus, observada no presente estudo, apresentou pequenas diferenças com aquelas encontradas para outras espécies do gênero presentes em regiões temperadas, como para $O$. strigicolllis, de $11,0^{\circ} \mathrm{C}$ para ninfas que originaram fêmeas e $10,6^{\circ} \mathrm{C}$ para machos, alimentados com ovos de A. kuehniella (Otha, 2002). Para O. sauteri, a temperatura base foi de $10,3^{\circ} \mathrm{C}$ (Yano et al., 2002) e para $O$. laevigatus, de $11,19^{\circ} \mathrm{C}$ (Alauzet et al., 1994). Também essas pequenas variações são inerentes a

Tabela III. Limite térmico inferior de desenvolvimento $(\mathrm{Tb})$, constante térmica $(\mathrm{K})$, equação linear da velocidade de desenvolvimento e coeficiente de determinação $\left(\mathrm{R}^{2}\right)$ para Orius insidiosus (Say).

\begin{tabular}{|c|c|c|c|c|c|}
\hline \multicolumn{2}{|c|}{$\begin{array}{c}\text { Fase de } \\
\text { desenvolvimento }\end{array}$} & $\mathrm{Tb}\left({ }^{\circ} \mathrm{C}\right)$ & $\mathrm{K}(\mathrm{GD})$ & Equações (1/D) & $\mathrm{R}^{2}(\%)$ \\
\hline \multicolumn{2}{|c|}{ Ovo } & 11,78 & & $-0,184763+0,015686 x$ & 99,71 \\
\hline \multirow{2}{*}{$1^{\circ}$ instar } & Macho & 11,87 & 32,62 & $-0,363780+0,030653 x$ & 93,23 \\
\hline & Fêmea & 11,85 & 32,03 & $-0,369854+0,031221 x$ & 97,15 \\
\hline \multirow{2}{*}{$2^{\circ}$ instar } & Macho & 11,40 & 29,09 & $-0,391883+0$ & 97,0 \\
\hline & Fêm & 11,42 & 28,88 & $-0,39$ & 96,63 \\
\hline \multirow{2}{*}{$3^{\circ}$ instar } & $\mathrm{Macl}$ & & & $-0,462908+0$ & \\
\hline & Fêm & 11,93 & & $-0,438091+0$ & 98,61 \\
\hline \multirow{2}{*}{$4^{\circ}$ instar } & $\mathrm{Mac}$ & & 24,52 & $10780 x$ & \\
\hline & Fêmea & 13,11 & 24,54 & $-0,534053+0,040752 x$ & 99,46 \\
\hline \multirow{2}{*}{$5^{\circ}$ instar } & Macho & & & $-0,259698+0,020623 x$ & 99,16 \\
\hline & Fêmea & 13,13 & 45,72 & $-0,287219+0,021874 x$ & 98,88 \\
\hline \multirow{2}{*}{$\begin{array}{l}\text { Fase } \\
\text { ninfal }\end{array}$} & Macho & 12,27 & 161,97 & $-0,075737+0,006174 x$ & 96,90 \\
\hline & Fêmea & 13,03 & 157,24 & $-0,079587+0,006360 x$ & 98,7 \\
\hline
\end{tabular}



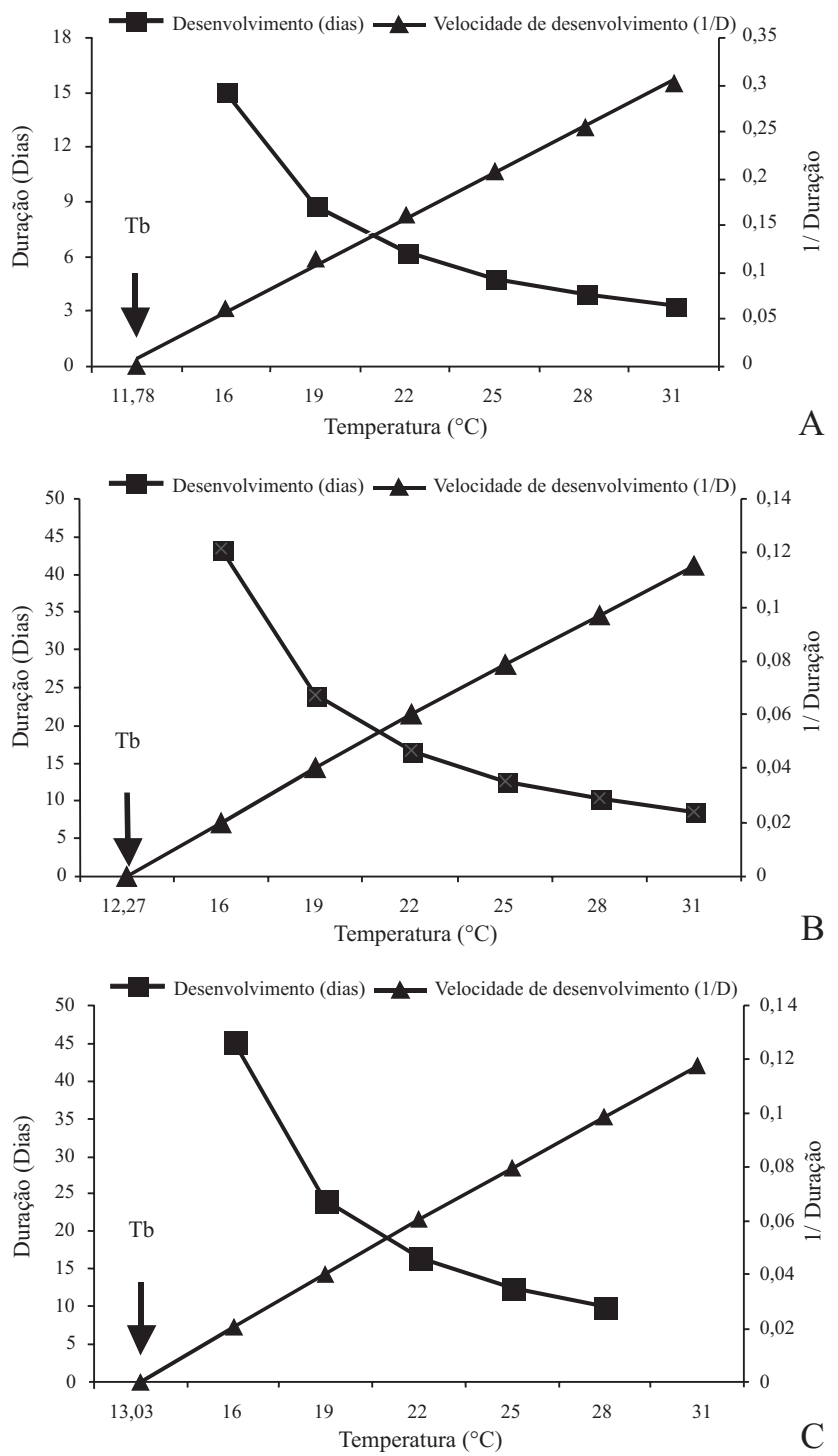

Fig. 1. Relação entre a temperatura, duração (dias) e a velocidade de desenvolvimento (1/D) das fases de ovo (A), e ninfal de machos (B) e de fêmeas (C) de Orius insidiosus (Say).

cada espécie e à capacidade das mesmas em explorar regiões climáticas diferentes.

Assim, o desenvolvimento e a sobrevivência de $O$. insidiosus, presente em área tropical, foram influenciados pelas diferentes temperaturas a que foi submetido. Conhecimentos básicos sobre as características do ciclo de vida do predador são requeridos para otimizar um sistema de criação massal, um importante passo no estabelecimento de um programa de controle biológico. Também as exigências térmicas, como a temperatura base e a constante térmica, são caracteres essenciais quando se busca o desenvolvimento do inseto em áreas de introdução e/ou liberação como agente de controle, possibilitando o entendimento de sua distribuição geográfica e sazonal.
Agradecimentos. Ao CNPq e a CAPES pela concessão de bolsas de estudos e a FAPEMIG pelo apoio financeiro.

\section{REFERÊNCIAS}

Alauzet, C.; D. Dargagnon, \& J. C. Malusa. 1994. Bionomics of a polyphagous predador: Orius laevigatus (Het. Anthocoridae). Enthomofaga, 39: 33-40.

Bueno, V. H. P. 2000. Desenvolvimento e multiplicação de percevejos predadores do gênero Orius Wolff. Cap. 6, p. 69-90. In: Bueno, V. H. P. (Ed.) Controle biológico de pragas: produção massal e controle de qualidade. Lavras: UFLA. 207p.

Chapman, R. F. 1998. Part III The abdomen, Reproduction and Developments. p. 259-408. In: The insects structure and function. Cambridge: University Press. 637p.

Dent, D. R. 1997. Quantifying insect populations: estimatives and parameters. p. 57-109. In: Dent, D. R.; Walton, M. P. (Ed.) Methods in ecological e agricultural entomology. Wallingford, UK: CAB International.

Haddad, M. L. \& J. R. P. Parra. 1984. Métodos para estimar exigências térmicas e os limites de desenvolvimento dos insetos. Piracicaba: FEALQ, 45 p.

Higley, S. G.; L. Pedigo \& K. R. Ostlie. 1986. DEGDAY: A program for calculating degree-days, and assumptions behind of degree-day approach. Environmental Entomology, 15: 999-1016.

Isenhour, D. J. \& K. V. Yeargan. 1981. Effect of temperature on the development of Orius insidiosus, with notes or laboratory rearing. Annals Entomological Society of America, 74: 114-116.

Lattin, J. D. 1999. Bionomics of the Anthocoridae. Annual Review of Entomology, 44: 207-231.

Mccfrey, J. P. \& R. L. Horsburgh. 1986. Functional response of Orius insidiosus (Hemiptera: Anthocoridae) to European Red Mite, Panonychus ulmi (Acari : Tetranychidae) at different temperatures constants. Environmental Entomology, 15: 532-535.

Mendes, S. M. \& V. H. P. Bueno. 2001. Biologia de Orius insidiosus (Say) (Hemiptera: Anthocoridae) alimentado com Caliothrips phaseoli (Hood) (Thysanoptera: Thripidae). Neotropical Entomology, 30: 423-428.

Mendes, S. M.; V. H. P. Bueno; V. M. Argolo \& L. C. P. Silveira, 2002. Type of prey influences biology and consumption rate of Orius insidiosus (Hemiptera: Anthocoridae). Revista Brasileira de Entomologia, 46: 99-103.

Messenger, P. S. 1959. Bioclimatic studies with insects. Annual Review of Entomology, 1: 183-206.

Otha, I. 2002. Effect of temperature on development of Orius strigicollis (Heteroptera: Anthocoridae) fed on Frankliniella occidentalis (Thysanoptera: Thripidae). Applied Entomological and Zoological, 36: 483-488.

Riudavets, J. 1995. Predators of Frankliniella occidentalis (Perg.) and Thrips tabaci Lind.: a review. p. 43-87. In: Loomans, A. J. M.; Van Lenteren J. C. (Ed.). Biological control of thrips pests. Wageningen: Wageningen: Agricultural University Papers.

Schmidt, J. M.; J. R. Taylor \& J. A. Rosenheim. 1998. Cannibalism and intraguild predation in the predatory Heteroptera. p. 133-169. In: Coll, M.; Ruberson, J. R. (Ed.) Predatory Heteroptera: their ecology and use in biological control. Lanham.

Scott, A. J. \& M. A. Knott. 1974. A cluster analyses method for grouping means in the analyses of variance. Biometrics, 30: 507-512.

Tommasini, M. G. \& G. Nicoli. 1994. Pre-imaginal activity of four Orius species reared on two preys. IOBC/WPRS Bulletin, 17: 237-241.

Van Den Meiracker, R. A. F. 1994. Induction and termination for diapause in Orius predatory bugs. Entomologia Experimentalis et Applicata, 73: 127-137.

Van Den Meiracker, R. A. F. 1999. Biocontrol of western flower thrips by heteropteran bugs. 145 p. Theis (PhD) - Amsterdan University, Amsterdam.

Yano, E.; K. Nagai; K. Watanable \& K. Yara. 2002. Biological parameters of Orius spp. for control of thrips in Japan. IOBC/ WPRS Bulletin, 25: 305-308. 